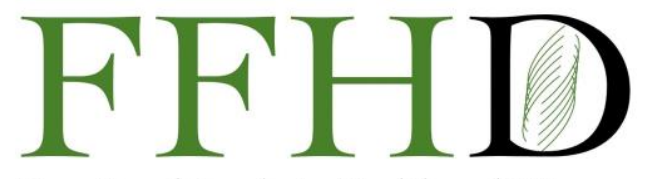

Functional Foods in Health and Disease

\title{
Absorption pathway of dietary flavonoids: the potential roles of the lymphatic transport in the intestine
}

\author{
Kaeko Murota \\ Department of Life Sciences, Faculty of Life and Environmental Sciences, Shimane University \\ 1060 Nishikawatsu-cho, Matsue, Shimane 690-8504 Japan
}

Corresponding Author: Kaeko Murota Department of Life Sciences, Faculty of Life and Environmental Sciences, Shimane University 1060 Nishikawatsu-cho, Matsue, Shimane 690-8504 Japan

Submission Date: June $17^{\text {th }}, 2020$; Acceptance Date: June $25^{\text {th }}, 2020$; Publication Date: July $15^{\text {th }}, 2020$

Please cite this article as: Murota K. Absorption pathway of dietary flavonoids: the potential roles of the lymphatic transport in the intestine Functional Foods in Health and Disease 2020; 10(7): 274-289. DOI: https://doi.org/10.31989/ffhd.v10i7.723

This article is a part of special issue of ICoFF/ISNFF 2019, Kobe, Japan. Special issue editors: Yasuhito Shirai, PhD, Professor, Graduate School of Agricultural Science, Department of Agrobioscience, Kobe University, Kobe, Japan and Hiroshi Yoshida, MD, PhD, Professor, The Jikei University School of Medicine, Tokyo, Japan 


\section{INTRODUCTION}

Flavonoids are one of the major phytochemicals in the diet and have attracted much attention to potentially prevent various diseases related to oxidative stress, such as atherosclerosis and coronary heart disease [14], as well as metabolic syndrome [5]. It is known that the bioavailability of flavonoids is an important factor determining their physiological functions in the body.

Flavonoids are present mainly in the form of glycosides in plant foods. After hydrolysis, their aglycone enters the intestinal epithelial cells and is metabolized by the phase II enzymes producing the corresponding conjugated metabolites [6, 7]. Following the intestinal conjugation, absorbed flavonoids are transported to either the portal vein or the lacteals. Most of the flavonoid studies focus on the metabolites in the plasma [8-11], while the potential of the lymphatic transport of dietary flavonoids has not been investigated until recently.

The small intestine is the primary tissue to absorb most dietary nutrients [12]. Carbohydrates are hydrolyzed with o-amylases, derived from the salivary gland and the pancreas, and several o-glucosidases at the intestinal mucosa, resulting in glucose, galactose and fructose as the absorbable monomers. Proteins are hydrolyzed by pepsin in the stomach, trypsin and other proteinases secreted from the pancreas, and finally free amino acids and small peptides are produced. These digestive products are hydrophilic and cross the intestinal epithelial monolayers via suitable membranous transporters, and in turn, they are transported into the blood capillaries under the epithelial layers as monosaccharides and amino acids, consequently reaching to the portal vein. In the case of dietary lipids, the lipophilic digestive products by various lipases, mainly secreted from the pancreas, are mixed with bile salts in the intestinal lumen to form the mixed micelle to make the hydrophobic molecules across the aqueous phase toward the intestinal mucosa. The major digestive products are free fatty acids and they are mostly resynthesized as triacylglycerol (TAG) and incorporated into the chylomicrons in the enterocyte. Chylomicrons are lipoprotein particles containing TAGs, phospholipids, cholesterol esters and fat-soluble vitamins with apolipoprotein B-48. Chylomicrons are transported into lymphatic capillaries called lacteals, and further through intestinal or mesenteric collecting lymphatic vessels to the thoracic duct. Chylomicrons finally flow into the systemic blood circulation via thoracic vein.

Recently, we found that dietary flavonoids are also transported into the lymph [13-15], although the mechanism and its physiological role are not clear. In this article, the general concept of the lymph transport of dietary components is summarized, and the potential of dietary flavonoid absorption pathway into the lymph is discussed.

Lymph as the absorption pathway of dietary lipids and lipophilic molecules

Lymphatic system in the intestine: The lymphatic system is a network of vessels in which the lymph fluid flows throughout the body [16]. Excess water effluxed from the blood vessel becomes the interstitial fluid, and is collected in the lymphatic vessel, eventually returning to the vein. This allows blood to maintain the volume and the levels of its component during circulation. Also, it is well known that the lymph system is essential in the immunological defense system, although such aspects are not described in this article.

The important role of the lymph system in the nutritional aspect is the absorption pathway of dietary lipids [17, 18]. Dietary lipids absorbed in the small intestine are taken up into the lymph vessels of the intestine as chylomicrons and transported to the vein through the thoracic duct. The villus of the small 
intestine possess both lymphatic and blood vessels inside and therefore dietary components that are absorbed across the small intestinal epithelium enter either lymphatic or blood capillaries. The lacteals are blunt-ended thin-walled lymphatic capillaries that are located in the middle of each villus. Chylomicrons are too large to enter the blood capillaries and transfer to the intestinal lymphatic capillaries called the lacteals, since the endothelial structure of the lacteals are significantly more permeable than the blood capillaries [17]. Chylomicrons are thought to reach the lacteal lumen by paracellular transport through the open intercellular junctions between lymphatic endothelial cells, whereas the transcellular transport mechanism has also been described [19].

\section{Intestinal absorption mechanism of dietary lipids:} Dietary fat is predominantly in the form of TAG. Dietary TAG is composed of various FAs esterified to a glycerol backbone. TAG is water insoluble and must be digested to be transferred across the aqueous fluid in the intestinal lumen to reach the intestinal epithelium for absorption. Dietary fat is digested in the beginning in the stomach with gastric lipases (and partly lingual lipase) to hydrolyze TAG to form mostly diacylglycerol (DAG) and free fatty acids [20]. Gastric lipase from human infants is reported to favor TAG with middlechain fatty acids and considered physiologically significant in newborns [21]. In the intestinal lumen, DAG and the rest of TAG are further hydrolyzed by pancreatic lipase. Pancreatic lipase possesses positional specificity against $s n-1$ and -3 positions of glycerol and forms 2-monoacylglycerol (2-MAG) and free fatty acids. In the case of phospholipids, which consist of approximately $5 \%$ of dietary lipids, pancreatic phospholipase $A_{2}$ is the main digestive enzyme to produce a free fatty acid and 1-acyl-lysophospholipid [22]. Cholesterol in the diet is often esterified with a long-chain fatty acid, and such ester bond is hydrolyzed with carboxyl ester lipase secreted from the pancreas [23]. Although carboxyl ester lipase was used as an enzyme to hydrolyze dietary retinyl esters, it has been found that pancreatic lipase is the main digestive enzyme for vitamin A ester [24].

The lipophilic digestive products are mixed with bile salts to form the mixed micelle, resulting in hydrophobic molecules that can diffuse the aqueous phase on the surface of the intestinal epithelium called the unstirred water layer. The mixed micelle has a hydrophilic outer surface with bile salts and phospholipids and a hydrophobic center. 2-MAG from lipolysis is solubilized within the center of the micelle because of its water-insolubility together with relatively hydrophobic fatty acids, cholesterol, and lipophilic vitamins. Mixed micelle presents the lipolytic products to the apical surface of the enterocyte. When the concentrations of the digestive products in the unstirred water layer fall below the critical micellar concentration, each monomer leaves the aggregation and diffuses toward the enterocyte. Classically, fatty acids and other lipophilic compounds are thought to enter the cell only via simple diffusion due to their affinity to the cellular membrane. Currently, several membranous proteins have been found as candidates of transporters for fatty acids [25-27], cholesterol [28], and fat-soluble vitamins [29-32].

Once in the enterocyte, the 2-MAG and FA migrate to the endoplasmic reticulum (ER). In the smooth ER, 2MAG and CoA-activated fatty acids (acyl-CoA) molecules are re-esterified to form TAG, which is called a MAG pathway predominantly used in the enterocyte. There is another pathway, called a phosphatidic acid pathway, to synthesize TAG from acyl-CoA and glycerophosphate. TAGs are consequently incorporated into the chylomicrons, along with other lipophilic compounds. 
Chylomicrons are lipoprotein particles that are synthesized in the enterocyte after lipid intake, with a hydrophilic outer surface consisting of phospholipids and apolipoproteins, and a hydrophobic core of TAG, cholesterol esters and other lipophilic molecules. They consist of approximately 80 to $90 \%$ TAG, 7 to $9 \%$ phospholipids, 1 to $5 \%$ cholesterol and its ester, and $2 \%$ proteins, mainly apolipoproteins B-48 (apo B-48) with A-I, A-IV and C.

TAG resynthesized in the smooth endoplasmic reticulum is incorporated into a lipid droplet coated with cholesterol, phospholipid, and apolipoproteins. Small particles containing apo B-48 and small amount of lipids are produced in the rough ER. This particle combines with TAG-rich particles from smooth ER, forming prechylomicrons. This particle is further processed in the Golgi apparatus to form the mature chylomicron, added apo A-I and A-IV. Finally, it is released by the enterocyte through exocytosis. Apo $C$ is later added from $\mathrm{HDL}$ in the circulation. Detailed mechanisms of the chylomicron assembly and its transport to the lymph are found in other excellent reviews $[17,18,33-36]$.

The role of lymphatic transport in absorption of lipophilic molecules: Lymphatic transport also plays an important role in the oral bioavailability of a number of highly lipophilic drugs and other xenobiotics [36].

Fatty acids with chain lengths of 14 or greater are more highly lymphatically transported, whereas middle and short chain fatty acids are primarily absorbed via the portal blood [37]. Therefore, long chain fatty acids and long chain TAGs more effectively support lymphatic drug transport compared to shorter chain fatty acids and their TAGs [38, 39]. Phosphatidylcholine (PC) and its digestion product, lysophosphatidylcholine (LPC) act as a surfactant in the mixed micelle in the intestinal lumen and thus enhance the absorption of lipophilic molecules. For example, LPC has been shown to enhance the intestinal absorption of carotenoids and the hydrolysis of PC is necessary to enhance the absorption of carotenoids [40-42]. Furthermore, it has been suggested that LPC uptake into the enterocyte is essential to assemble the chylomicron in the enterocyte [43], which is an important step to absorb the lipophilic molecules.

Due to the unique anatomy and physiology of the lymphatics, intestinal lymphatic drug transport can provide many advantages over drug absorption via the portal blood [36]. Absorption from the lymph is generally known as a route to avoid detoxification metabolism in the liver. Metabolites with a low degree of conjugation show a relatively high activity. Drugs that are absorbed via the intestinal lymphatic system are essentially protected from hepatic first-pass metabolism since the mesenteric lymph enters the systemic circulation directly. Also, drug transport via the intestinal lymph may result in the direct drug exposure to the lymphatics and a changed mode of delivery to the systemic circulation and the peripheral tissues.

\section{Lymphatic absorption pathway of dietary flavonoids} General aspect of dietary flavonoid absorption: The absorption and metabolism of dietary flavonoids have been studied more and more actively in recent years, and the plasma profile after their consumption has been widely investigated [8-11, 44]. Among various flavonoids, quercetin is one of the major molecules in plant foods and its bioavailability has been well investigated. Quercetin is found as its various glycosides such as quercetin 3-O-glucoside (Q3G or isoquercitrin), quercetin $4^{\prime}-O$-glucoside $\left(\mathrm{Q}^{\prime} \mathrm{G}\right)$, quercetin $3,4^{\prime}-\mathrm{O}$ diglucoside $\left(Q 3,4^{\prime} G\right)$, and rutin (quercetin 3-Orutinoside) in vegetables and fruits. The chemical structures of flavonoids featured in this article are 
found in Figure 1. Most flavonoid glycosides are not absorbed directly, and deglycosylation is an essential step [6]. Flavonoid glycosides are hydrolyzed to their corresponding aglycone either by lactase-phlorizin hydrolase (LPH) existing in the intestinal mucosa [45, 46] or intestinal bacteria. Monoglucosides such as Q4'G are partly taken up via sodium glucose co-transporter type 1 (SGLT-1) [47], and then converted into aglycones by cytosolic $\beta$-glucosidase (CBG) $[48,49]$. However, the glucosides taken up directly are easily excreted to the luminal side via MRP2 protein [50].

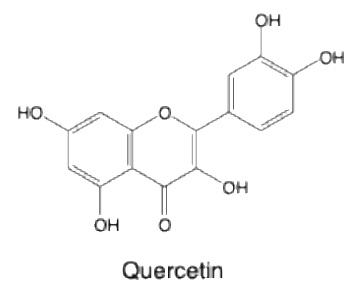

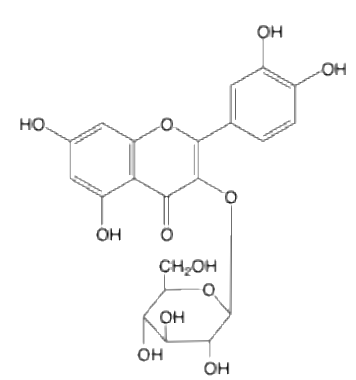

Quercetin 3-O-glucoside (Q3G)

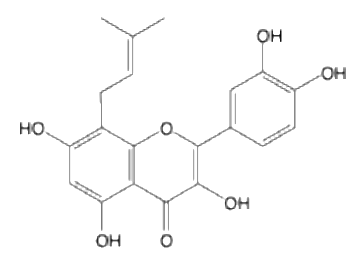

8-prenyl quercetin

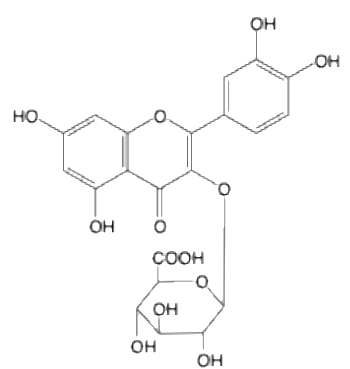

Quercetin 3-O-glucuronide

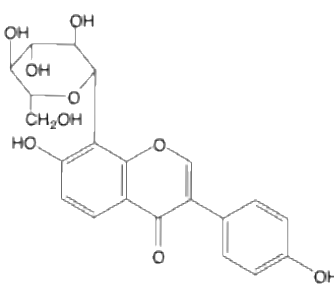

Puerarin (Daidzein 8-C-glucoside)
Fig. 1 Chemical structures of flavonoids

Representative molecules with different modifications are shown.

Considerable flavonoids have an affinity to the cell membrane and favor the oil-water interface [51, 52].
The aglycone produced after digestion is generally thought to enter the enterocyte via simple diffusion pathway, whereas several membranous proteins are reported as candidates for certain flavonoid molecules $[53,54]$. The aglycone in the enterocyte is then metabolized by the intracellular phase II enzymes, such as UDP-glucuronosyltransferase (UGT), sulfotransferase (SULT), and catechol-O-methyltransferase (COMT) [55]. In the small intestine of a rat, uridine-5'-diphosphateglucuronosyltransferases (UGT) is considered as a major enzyme that conjugates flavonoids with glucuronic acid $[55,56]$. In humans, both UGT and SULT are considered to contribute to the production of monoglucuronides and sulfates $[8,55]$.

Other flavonoid glycosides to have diglycoside or monoglycoside other than glucose attached are transferred to the distal intestine and assimilated by the intestinal microbiota. They hydrolyze various flavonoid aglycone and further catabolize them to yield degraded phenolic acids and reduced catabolites [57]. The epithelial cells in the colon can absorb the aglycones and their catabolites, and produce their phase II conjugated metabolites within the cell [56].

The intestinal metabolites are further metabolized in the liver and undergo multiple conjugation [8]. These metabolites are partly excreted via the bile from the liver to the small intestinal lumen [58]. The conjugated metabolites excreted in the small intestine are deconjugated by the action of intestinal bacteria and are again absorbed from the large intestine, which is termed as the enterohepatic circulation. The enterohepatic circulation is considered to contribute to the prolongation of the retention time in the body and the increase in the accumulated amount $[58,59]$. Most various metabolites circulate in blood as highly watersoluble conjugates and are eventually excreted in urine. 
Lymphatic transport of quercetin and its glycosides: As mentioned above, the gastrointestinal lymph generally plays an important role in the absorption of dietary lipids and other lipophilic compounds. However, little is known about the significance of the lymphatic pathway in the absorption of amphiphilic dietary compounds.

We have reported for the first time that quercetin was partly transported into the thoracic lymph as its conjugated metabolites after administration of its aglycone in a study of unanesthetized thoracic lymphcannulated rats in 2005 [13]. The intestinal lymph systems following the lacteals are gathered to form a larger collecting lymph duct and reach the thoracic lymph duct, and thus, the thoracic lymph-cannulated rat model is widely used for estimating the absorption of various lipids and other lipophilic compounds [39, 60-62]. Cannulation surgery is performed under anesthesia the day before the sample administration and the rats are conscious during experiment. In our first report [13], quercetin aglycone was administered as propylene glycol mixture into the stomach of rats. Quercetin were rapidly absorbed and reached maximal levels in the lymph within $30 \mathrm{~min}$. Interestingly, the lymphatic quercetin was mostly detected in hydrophilic conjugated forms. The intact aglycone that is more hydrophobic only detected within $1 \mathrm{~h}$ after administration, and its levels were much lower than the conjugated metabolites. Methylated conjugate metabolites were also detected, but did not show the apparent absorption peak.

We further investigated the lymphatic transport of Q3G and compared it to quercetin aglycone [15]. The total concentration of quercetin in the lymph (obtained after deconjugation treatment) was about four times lower than that in the plasma after administration of both Q3G and the aglycone, and not significantly different between two groups. Moreover, quercetin aglycone was detected only in the lymph of a few of rats at $0.5 \mathrm{~h}$ after administration of Q3G and the aglycone.
Q3G was also detected in the lymph from all rats $0.5 \mathrm{~h}$ after administration of Q3G and the aglycone, and it was detected at slightly higher levels after Q3G administration than after the aglycone administration, although the difference was not significant $(p=0.058)$. This data suggests that only small amounts of the intact aglycone and the glucoside are absorbed right after administration and transported directly into the lymph after administration of the aglycone and Q3G, whereas the majority of the absorbed quercetin is extensively metabolized in the enterocyte. Even if the aglycone was transported via the portal vein, the hepatocyte would rapidly metabolize it and would not pass through the liver without conjugation. Interestingly, Q3G appeared in the lymph after administering the aglycone as well as Q3G itself. There is a report that shows microsomes from the liver, kidney, and intestinal mucosa were able to catalyze the formation of glucoside conjugates of bile salt [63], although glucosidic Q metabolites were only found in the urine but not in the plasma [8]. Thus, our data suggests that the glucoside conjugates might be produced in the small intestine and only transported in the lymph because the lymphatic system is used to maintain the body's fluid by removing dispensable products from the interstitial tissue.

Chen et al. [64] reported the lymphatic transport of intact quercetin and its glycoside rutin in rats. They found that the lymphatic concentration of quercetin aglycone was higher than in the plasma. In the case of rutin, the dose of 10 times higher than quercetin resulted in the appearance of rutin itself in the lymph, and the level was similar to that of the plasma. Although the metabolites were not determined and the whole profile of their absorption were not clarified in the study, it is suggested that more hydrophobic aglycone could be predominantly transported into the lymph. 
The localization of quercetin metabolites in the lymph:

In general, highly lipophilic drugs (typically with $\log \mathrm{P}>$ 5) are easily incorporated into developing chylomicrons in the enterocyte and access to the intestinal lymph [36]. $\log P$ is generally defined as the logarithm of the partition coefficient in octanol/water suspension at a defined temperature. The flavonoid aglycone produced after the hydrolysis of sugar moieties in the digestive tract becomes less hydrophilic and insoluble in water compared to its glycosidic forms, confirmed by Rothwell et al. [65] Quercetin aglycone has a log P value of around 1.8 [66] while its glycosides and the conjugate metabolites produced in the body show smaller log $\mathrm{P}$ [51] (Table 1). Therefore, it is not likely that the flavonoids could be incorporated into the chylomicrons' formation.

Quercetin absorption is known to be enhanced when it is co-administered with lipids [67-70]. When quercetin was mixed with long-chain fatty acid TAG (soybean oil) and administered to the duodenum of lymph-cannulated rats, the lymph transport of quercetin metabolites was significantly promoted along with an increase in lymph triglyceride, compared to coadministration of triglycerides of medium-chain fatty acids [14]. Acceleration of absorption was also accompanied by an increase in blood flavonoid concentration. Interestingly, quercetin metabolites in lymph were not associated with chylomicron. The lymph obtained after quercetin administration together with soybean oil was centrifuged and the chylomicron was removed using its very low density. The concentrations of quercetin metabolites in the whole lymph and in the chylomicron removed fluid were almost the same, indicating that quercetin metabolites are solubilized in the lymph fluid but not as a part of chylomicrons. This observation is in accordance with our human trial data that most of quercetin metabolites in human plasma localized in the lipoprotein deprived fraction in which serum albumin is the major component [71].

Quercetin has a coplanar conformation and has a strong affinity to the oil-water interface. Such characteristics are kept even if the aglycone is conjugated with sugars. Shirai et al. [72] measured the affinity of quercetin and its related compounds to the large unilamellar liposomal membranes prepared with egg yolk lecithins. Quercetin aglycone showed the binding ability to the liposomes, and its affinity was intermediate between that of lipophilic a -tocopherol and that of hydrophilic ascorbic acid. The affinities to the liposome of Q3G and quercetin 3-O-glucuronide were lower than those of its aglycone, but significantly higher than that of ascorbic acid. This observation means that the glucoside and the glucuronide of quercetin are less lipophilic than its aglycone but possess significant affinity to the lipid membrane (Table 1). Thus, it is more likely that quercetin in the lymph may be weakly associated with the chylomicrons at the interface, although this point needs further investigation.

Table 1. Lipophilicity and the affinity to the liposomal membrane of quercetin and its related compounds.

\begin{tabular}{lcc}
\hline & Log P & $\begin{array}{c}\text { Affinity to the } \\
\text { liposomal } \\
\text { membrane }\end{array}$ \\
\hline Quercetin & $1.82^{\mathrm{a}, \mathrm{b}}$ & $0.95^{\mathrm{f}}$ \\
Quercetin 3-O- & $0.69^{\mathrm{a}}$, & $0.37^{\mathrm{f}}$ \\
glucoside & $0.76^{\mathrm{b}}$ & \\
Quercetin 3-O- & $-2.1^{\mathrm{c}}$ & $0.36^{\mathrm{f}}$ \\
glucuronide & & \\
Q-Tocopherol & $12.2^{\mathrm{d}}$ & $1.0^{\mathrm{f}}$ \\
Ascorbic acid & $-1.85^{\mathrm{e}}$ & $0^{\mathrm{f}}$ \\
\hline
\end{tabular}

aThe values are calculated from the data of the partition coefficient in octanol/water reported in [66]. 
bThe values are referred from [65].

cThe values are calculated from the data of the partition coefficient in octanol/water reported in [51].

dThe values are obtained from the PubChem database, [https://pubchem.ncbi.nlm.nih.gov/compount/21163\#sectio $\mathrm{n}=$ Octanol-Water-Partition-Coefficient\&fullscreen=true], retrieved June 15, 2020.

e The values are obtained from the PubChem database, [https://pubchem.ncbi.nlm.nih.gov/compount/54670067\#se ction=Octanol-Water-Partition-Coefficient\&fullscreen=true], retrieved June 15, 2020.

fThe values are calculated from the data reported in [51] as following:

[Concentration in the initial suspension] - ([Concentration in the filtrate after ultrafiltration of liposome suspension] / [Concentration in the initial suspension]).

\section{Chemical structure of quercetin metabolites in the} lymph: In earlier studies, the bioavailability of flavonoids was investigated by measuring the total amounts of quercetin in the lymph and in the plasma after deconjugation treatment $[13,14,71]$. Today, it is well recognized that the physiological function of dietary flavonoids largely depends on the chemical structure of their metabolites in the body. Thus, the analysis of the chemical structure of flavonoid metabolites in the plasma and in the urine has been widely performed using LC-MS/MS $[8,11]$. Compared with such metabolome studies in the plasma and in the urine, the lymphatic profile of flavonoids remains to be determined. We first reported the metabolites of quercetin in the lymph and in the plasma after administering Q3G and its aglycone [15]. In the lymph, we found both primary conjugated metabolites produced in the small intestine which may transfer directly to the lymph, and the secondary conjugated metabolites produced in the liver, the latter may have been transferred from the peripheral circulation (Table 2).

Multiple conjugates that are produced in the hepatocyte appeared in the lymph later than in the plasma and constituted a relatively large portion at later time points, suggesting that hepatic metabolites are transferred from the peripheral plasma to the lymph circulation.

This observation is in good agreement with the previous report by Labrid [73]. $\left[{ }^{14} \mathrm{C}\right]$-labeled diosmin,

Table 2. Quercetin metabolites detected in the lymph and in the plasm of thoracic lymph-cannulated rats.

\begin{tabular}{|c|c|c|}
\hline & $\begin{array}{l}\text { Differences between } \\
\text { in the lymph }\end{array}$ & the lymph and the plasma at $\mathrm{T}_{\max }$ \\
\hline & Q3G administration & Aglycone administration \\
\hline \multicolumn{3}{|c|}{$\mathrm{T}_{\max }$ in the lymph $=0.5 \mathrm{~h}$ after administration } \\
\hline Quercetin monoglucuronides & $P<0.05$ & $P<0.05$ \\
\hline Isorhamnetin monoglucuronides & $P<0.05$ & N.S. \\
\hline Quercetin monosulfates & $P<0.05$ & N.S. \\
\hline Isorhamnetin monosulfates & $P<0.05$ & $P<0.05$ \\
\hline \multicolumn{3}{|c|}{$\mathrm{T}_{\max }$ in the lymph $=1.0 \mathrm{~h}$ after administration } \\
\hline Quercetin diglucuronides & N.S. & $P<0.05$ \\
\hline Isorhamnetin diglucuronides & N.S. & $P<0.05$ \\
\hline
\end{tabular}

When the differences are significant, the plasma levels are higher than the lymph levels. N.S., not significant ( $>>0.05)$. The original results are found in [15] 
which is a major component of Daflon ${ }^{\circledR} 500 \mathrm{mg}$, was administered by intravenous injection to the anesthetized dogs and rats with thoracic duct fistula, and the radioactivity was detected in the lymph showing the blood-to-lymph transfer of this flavonoid.

Thus, dietary flavonoids can reach the lymph circulation through two steps: direct transport from the intestine, and then the circulating flavonoid metabolites transfer from blood to the lymph (Fig. 2).

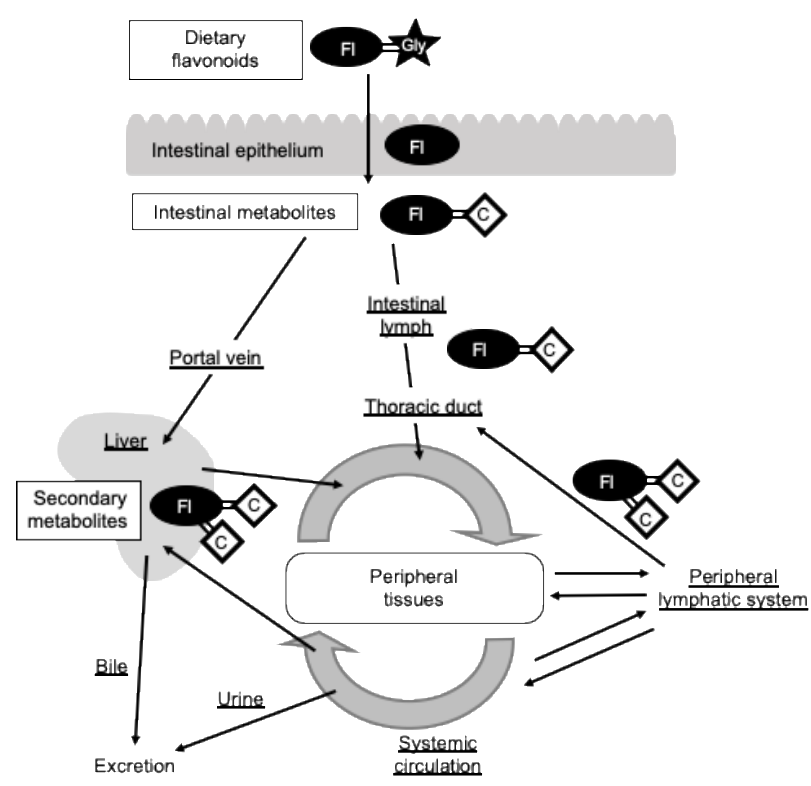

Fig. 2 Putative intestinal absorption pathway of dietary flavonoids.

Flavonoid glycosides are taken up into the enterocyte after hydrolysis of sugar moiety and are converted into the conjugated metabolites. The intestinal metabolites are transported either via the portal vein or via the intestinal lymph duct. The former pathway brings the metabolites into the liver and the secondary metabolism occurs. The latter pathway leads the intestinal metabolites directly into the systemic circulation. The hepatic secondary metabolites are transported into the systemic circulation, and are possibly transferred into the peripheral lymphatic system. C, conjugation group; Gly, glycosidic moiety; FI, flavonoid aglycone.

Interestingly, the profile patterns of the conjugated metabolites after Q3G and the aglycone administration were different in the lymph and in the plasma [15]. When Q3G was administered, the deglycosylation step by lactase-phlorizin hydrolase yielded its aglycone gradually, and the rate of the uptake into the enterocyte may modulate the selectivity of metabolic pathways between UGT and SULT and in turn the favorable transport direction between toward the lacteals and the blood capillary.

Factors affecting lymphatic transport of dietary

flavonoid: Similarly to lipophilic drugs, the type of coadministrated lipids affects the lymphatic transport of flavonoids. In our study, soybean oil is better than middle-chain fatty acyl TAG at enhancing the quercetin absorption [14]. Makino et al. also reported similar results, and further showed that high-fat diet exposure prior to quercetin administration showed additional enhancing effect [74].

Several groups use the nanoemulsion particle to enhance the medicinal flavonoid absorption. Puerarin is the 8 -C-glucoside of daidzein and found in Pueraria labota, called "kuzu" in Japan. This plant medicine is traditional used in Chinese medicine and is expected to prevent stroke. Flavonoid C-glycoside is known to be poorly absorbed in the gastrointestinal tract. The potential for microemulsion drug delivery systems of a poorly water-soluble drug, puerarin, were investigated in anesthetized lymph-cannulated rats $[75,76]$. Zhou et al. used anesthetized lymph-cannulated rats to compared to non-lymph-cannulated rats [76]. Their results showed that nanoemulsion, especially lipidbased nanosuspension, effectively enhances the bioavailability of puerarin, mainly via lymphatic transport. 
The other experimental system is used to estimate the possibility of the lymphatic transport of flavonoids. $\mathrm{Xu}$ et al. suggest that nanoemulsion of baicalin (baicalain 7-O-glucuronide) was absorbed more than simple suspension using the chylomicron flow blocking model [77]. In another case, using human Caco-2 cells, it was shown that 5-hydroxy-6,7,8,3',4'pentamethoxylflavone (5-HPMF) were taken up more with oleic acid than the control to enhance the incorporation into chylomicron [78]. Polymethoxyflavonoid shows relatively high lipophilicity among flavonoids and is more likely to be a component of chylomicron, although Caco-2 cells can synthesize chylomicron much less than in vivo enterocyte.

Prenylated flavonoids possess a C5 isoprenyl unit in a diphenylpropane structure, and the prenylation increases the lipophilicity of flavonoids. Mukai et al. [79] reported the bioavailability of 8-prenyl quercetin (8-PQ). Administrated 8-PQ was detected with deconjugation treatment in the plasma of mice as well as in the lymph of unanesthetized lymph-cannulated rats. Prenylation significantly enhanced the cellular uptake by increasing the lipophilicity and attenuated efflux from the cell, suggesting that prenylation enhances the accumulation of quercetin in tissue, although the apparent absorption from the intestine was lower than non-prenylated quercetin. The $\mathrm{C}_{\max }$ and AUC both in the plasma and the lymph was higher after quercetin administration than 8-PQ administration. However, the difference of the levels between the plasma and the lymph was smaller with 8-PQ than quercetin, suggesting that prenylation was effective in enhancing the lymphatic transport.

Potential roles of the lymphatic transport of dietary flavonoids: Lymphatic transport in the intestine is the essential pathway of dietary lipid absorption, and it provides some advantages to lipophilic drugs, such as avoiding the first-pass metabolism in the liver and modifying distribution to peripheral tissues. The conjugated metabolites generally show lower activity than their aglycones, and the activation mechanism by converting the glucuronide to the corresponding aglycone by the b-glucuronidase secreted at the target site of inflammation has been reported [80-82]. The presence of intact aglycone in the lymph, even though its level is very low, may have the potential that more active aglycone would be delivered to the target tissues. Moreover, there are many studies that indicate the effect of flavonoids on the immune system [83-85], and indicate intestinal lymphatic transport may play an important role in the delivery of flavonoids to the lymph nodes and lymphocytes.

In 1936, Szent-Gyorgi et al. first claimed that citrus flavonoids (hesperidin and rutin) reduced capillary fragility and permeability in human blood vessels [86]. At present, it is generally considered that flavonoids exert their action on the vascular endothelium. Daflon ${ }^{\circledR}$ $500 \mathrm{mg}$ is a flavonoid medicine (diosmin 90\% + hesperidin $10 \%$ ) that is used to alleviate perivascular edema. Using the thoracic lymph fistula of a dog, the effect of Daflon $^{\circledR} 500$ mg was investigated [73]. Intravenous injection of Daflon ${ }^{\circledR} 500$ mg enhanced the lymphatic flow, and diosmin (or its metabolites) was detected in the lymph circulation. The activity of flavonoids against the perivascular edema could be exerted via the lymphatic circulation.

At present, to the best of my knowledge, the lymphatic transport of dietary flavonoids has not been the region of interest in any human trial. Research progress in this field would be expected.

\section{CONCLUSION}

Understanding of the mechanism of action of dietary flavonoids has been increasing with the progress of 
their bioavailability research. We have reported that the lymph transport is the alternative absorption pathway of quercetin in the intestine. The chemical structure of quercetin in the lymph is mainly its conjugated metabolites, which are highly hydrophilic. The lymphatic transport was significantly promoted with the long-chain TAG co-administration along with an increase in lymph triglyceride, compared to coadministration of medium-chain TAG. Interestingly, quercetin metabolites are solubilized in the lymph fluid but not incorporated into the chylomicrons.

In the lymph, we found both primary conjugated metabolites produced in the small intestine which may transfer directly to the lymph, and the secondary conjugated metabolites produced in the liver, the latter may have been transferred from the peripheral circulation. These findings will be useful to clarify the mechanism of action of dietary flavonoids as functional food factors as well as exploring the new flavonoid compounds for novel medicinal drugs.

The physiological role of these lymphatic metabolites is unknown at present. From our results, it is not likely to protect the chylomicron from oxidation, the postulated mechanism of anti-atherosclerosis to prevent the LDL oxidation. The potential to deliver less conjugated molecules to the target tissues will be the next step to be clarified, such as the effect against edema and on the immune system.

At present, we only investigated the conjugated metabolites of flavonoids. However, recent interest has moved to the ring-fission catabolites that are generated by the intestinal microbiota [57]. The metabolomic analysis on such catabolites has been performed but only for in the plasma and in the urine [8-10]. Further investigation is necessary to estimate the catabolic molecules produced by the intestinal microbiota and their absorption pathway.
List of Abbreviations: CBG, cytosolic ?-glucosidase; COMT, catechol-O-methyltransferase; ER, endoplasmic reticulum; LPH, lactase phloridin hydrolase; MAG, monoacylglycerol; TAG, triacylglycerol; PC, phosphaticylcholine; 8-PQ, 8-prenyl quercetin; Q3G, quercetin 3-O-glucoside; SGLT-1, sodium glucose cotransporter type 1; UGT, uridine-5'-diphosphateglucuronosyltransferase

Competing interests: The author has no conflicts of interest to declare.

Author's contribution: Kaeko Murota drafted and revised this manuscript, and had responsibility for the final content.

Acknowledgments and funding: The research by KM et al. based on this work was partially supported by JSPS KAKENHI Grant Number 40294681

\section{REFERENCES}

1. Hertog MG, Feskens EJ, Hollman PC, Katan MB, Kromhout D: Dietary antioxidant flavonoids and risk of coronary heart disease: The Zutphen elderly study. Lancet 1993, 342:10071011

2. Knekt P, Jarvinen $R$, Reunanen A, Maatela J: Flavonoid intake and coronary mortality in finland: A cohort study. BMJ 1996, $312: 478-481$

3. Manach C, Mazur A, Scalbert A: Polyphenols and prevention of cardiovascular diseases. Curr Opin Lipidol 2005, 16:77-84.

4. Siasos G, Tousoulis D, Tsigkou V, Kokkou E, Oikonomou E, Vavuranakis $\mathrm{M}$ et al.: Flavonoids in atherosclerosis: An overview of their mechanisms of action. Curr Med Chem 2013, 20:2641-2660

5. Galleano M, Calabro V, Prince PD, Litterio MC, Piotrkowski B, Vazquez-Prieto MA et al.: Flavonoids and metabolic syndrome. Ann N Y Acad Sci 2012, 1259:87-94.

6. Murota K, Terao J: Antioxidative flavonoid quercetin: 
Implication of its intestinal absorption and metabolism. Arch Biochem Biophys 2003, 417:12-17.

7. Manach C, Scalbert A, Morand C, Remesy C, Jimenez L: Polyphenols: Food sources and bioavailability. Am J Clin Nutr 2004, 79:727-47.

8. Mullen W, Edwards CA, Crozier A: Absorption, excretion and metabolite profiling of methyl-, glucuronyl-, glucosyl- and sulpho-conjugates of quercetin in human plasma and urine after ingestion of onions. Br J Nutr 2006, 96:107-116.

9. Hosoda K, Furuta T, Yokokawa A, Ogura K, Hiratsuka A, Ishii K: Plasma profiling of intact isoflavone metabolites by highperformance liquid chromatography and mass spectrometric identification of flavone glycosides daidzin and genistin in human plasma after administration of kinako. Drug Metab Dispos 2008, 36:1485-95.

10. Crozier A, Del Rio D, Clifford MN: Bioavailability of dietary flavonoids and phenolic compounds. Mol Aspects Med 2010, 31:446-467.

11. Nakamura T, Murota K, Kumamoto S, Misumi K, Bando N, Ikushiro S et al.: Plasma metabolites of dietary flavonoids after combination meal consumption with onion and tofu in humans. Mol Nutr Food Res 2014, 58:310-7.

12. Johnson LR: Digestion and absorption of nutrients. In Gastrointestinal physiology (8th edition). Edited by Johnson LR. Philadelphia, PA: Elsevier Mosby; 2014:108-127.

13. Murota K, Terao J: Quercetin appears in the lymph of unanesthetized rats as its phase ii metabolites after administered into the stomach. FEBS Lett 2005, 579:53435346.

14. Murota K, Cermak R, Terao J, Wolffram S: Influence of fatty acid patterns on the intestinal absorption pathway of quercetin in thoracic lymph duct-cannulated rats. Br J Nutr 2013, 109:2147-53.

15. Nakamura T, Kinjo C, Nakamura $Y$, Kato $Y$, Nishikawa $M$, Hamada $\mathrm{M}$ et al.: Lymphatic metabolites of quercetin after intestinal administration of quercetin-3-glucoside and its aglycone in rats. Arch Biochem Biophys 2018, 645:126-136.

16. Moore Jr. JE, Bertram CD: Lymphatic system flows. Annual
Review of Fluid Mechanics 2018, 50:459-482.

17. Cifarelli V, Eichmann A: The intestinal lymphatic system:

Functions and metabolic implications. Cell Mol Gastroenterol Hepatol 2019, 7:503-513.

18. Hokkanen K, Tirronen A, Yla-Herttuala S: Intestinal lymphatic vessels and their role in chylomicron absorption and lipid homeostasis. Curr Opin Lipidol 2019, 30:370-376.

19. Dixon JB: Mechanisms of chylomicron uptake into lacteals. Annals of the New York Academy of Sciences 2010, 1207:E52-E57.

20. Rogalska E, Ransac S, Verger R: Stereoselectivity of lipases. Ii. Stereoselective hydrolysis of triglycerides by gastric and pancreatic lipases. J Biol Chem 1990, 265:20271-6.

21. Jensen RG, deJong FA, Lambert-Davis LG, Hamosh M: Fatty acid and positional selectivities of gastric lipase from premature human infants: In vitro studies. Lipids 1994, 29:433-5.

22. Nilsson A, Duan RD: Pancreatic and mucosal enzymes in choline phospholipid digestion. Am J Physiol Gastrointest Liver Physiol 2019, 316:G425-G445.

23. Weng W, Li L, van Bennekum AM, Potter SH, Harrison EH, Blaner WS et al.: Intestinal absorption of dietary cholesteryl ester is decreased but retinyl ester absorption is normal in carboxyl ester lipase knockout mice. Biochemistry 1999, 38:4143-9.

24. Harrison EH: Mechanisms of digestion and absorption of dietary vitamin a. Annu Rev Nutr 2005, 25:87-103.

25. Stremmel W: Uptake of fatty acids by jejunal mucosal cells is mediated by a fatty acid binding membrane protein. J Clin Invest 1988, 82:2001-10.

26. Poirier H, Degrace P, Niot I, Bernard A, Besnard P: Localization and regulation of the putative membrane fattyacid transporter (fat) in the small intestine. Comparison with fatty acid-binding proteins (fabp). Eur J Biochem 1996, 238:368-73.

27. Stahl A, Hirsch DJ, Gimeno RE, Punreddy S, Ge P, Watson N et al.: Identification of the major intestinal fatty acid transport protein. Mol Cell 1999, 4:299-308. 
28. Altmann SW, Davis HR, Jr., Zhu LJ, Yao X, Hoos LM, Tetzloff G et al.: Niemann-pick c1 like 1 protein is critical for intestinal cholesterol absorption. Science 2004, 303:1201-4.

29. During A, Dawson HD, Harrison EH: Carotenoid transport is decreased and expression of the lipid transporters sr-bi, npc1l1, and abca1 is downregulated in caco-2 cells treated with ezetimibe. J Nutr 2005, 135:2305-12.

30. Narushima K, Takada T, Yamanashi Y, Suzuki H: Niemann-pick c1-like 1 mediates alpha-tocopherol transport. Mol Pharmacol 2008, 74:42-9.

31. Reboul E, Goncalves A, Comera C, Bott R, Nowicki M, Landrier JF et al.: Vitamin d intestinal absorption is not a simple passive diffusion: Evidences for involvement of cholesterol transporters. Mol Nutr Food Res 2011, 55:691702.

32. Goncalves A, Margier M, Roi S, Collet X, Niot I, Goupy P et al.: Intestinal scavenger receptors are involved in vitamin k1 absorption. J Biol Chem 2014, 289:30743-52.

33. Hussain MM, Kancha RK, Zhou Z, Luchoomun J, Zu H, Bakillah A: Chylomicron assembly and catabolism: Role of apolipoproteins and receptors. Biochimica et biophysica acta 1996, 1300:151-170.

34. Kohan A, Yoder S, Tso P: Lymphatics in intestinal transport of nutrients and gastrointestinal hormones. Annals of the New York Academy of Sciences 2010, 1207:E44-E51.

35. Xiao C, Stahel P, Lewis GF: Regulation of chylomicron secretion: Focus on post-assembly mechanisms. Cellular and molecular gastroenterology and hepatology 2019, 7:487501.

36. Porter CJ, Trevaskis NL, Charman WN: Lipids and lipid-based formulations: Optimizing the oral delivery of lipophilic drugs. Nat Rev Drug Discov 2007, 6:231-48.

37. Harkins RW, Sarett HP: Medium-chain triglycerides. Jama $1968,203: 272-4$

38. Behrens D, Fricker R, Bodoky A, Drewe J, Harder F, Heberer M: Comparison of cyclosporin a absorption from lct and mct solutions following intrajejunal administration in conscious dogs. J Pharm Sci 1996, 85:666-8.
39. Caliph SM, Charman WN, Porter CJ: Effect of short-, medium-, and long-chain fatty acid-based vehicles on the absolute oral bioavailability and intestinal lymphatic transport of halofantrine and assessment of mass balance in lymphcannulated and non-cannulated rats. J Pharm Sci 2000, 89:1073-84.

40. Sugawara $T$, Kushiro $M$, Zhang $H$, Nara $E$, Ono $H$, Nagao $A$ : Lysophosphatidylcholine enhances carotenoid uptake from mixed micelles by caco-2 human intestinal cells. J Nutr 2001, 131:2921-7.

41. Baskaran V, Sugawara T, Nagao A: Phospholipids affect the intestinal absorption of carotenoids in mice. Lipids 2003, $38: 705-11$

42. Lakshminarayana R, Raju M, Krishnakantha TP, Baskaran V: Enhanced lutein bioavailability by lyso-phosphatidylcholine in rats. Mol Cell Biochem 2006, 281:103-10.

43. Siddiqi S, Mansbach $\mathrm{CM}$, 2nd: Dietary and biliary phosphatidylcholine activates pkczeta in rat intestine. J Lipid Res 2015, 56:859-70.

44. Loke WM, Jenner AM, Proudfoot JM, McKinley AJ, Hodgson JM, Halliwell B et al.: A metabolite profiling approach to identify biomarkers of flavonoid intake in humans. J Nutr 2009, 139:2309-14.

45. Day AJ, Canada FJ, Diaz JC, Kroon PA, McLauchlan R, Faulds CB et al.: Dietary flavonoid and isoflavone glycosides are hydrolysed by the lactase site of lactase phlorizin hydrolase. FEBS Lett 2000, 468:166-170.

46. Day AJ, Gee JM, DuPont MS, Johnson IT, Williamson G: Absorption of quercetin-3-glucoside and quercetin-4'glucoside in the rat small intestine: The role of lactase phlorizin hydrolase and the sodium-dependent glucose transporter. Biochem Pharmacol 2003, 65:1199-1206.

47. Walgren RA, Lin JT, Kinne RK, Walle T: Cellular uptake of dietary flavonoid quercetin 4'-beta-glucoside by sodiumdependent glucose transporter sglt1. J Pharmacol Exp Ther 2000, 294:837-843.

48. Day AJ, DuPont MS, Ridley S, Rhodes M, Rhodes MJ, Morgan MR et al.: Deglycosylation of flavonoid and isoflavonoid 
glycosides by human small intestine and liver betaglucosidase activity. FEBS Lett 1998, 436:71-75.

49. Nemeth K, Plumb GW, Berrin JG, Juge N, Jacob R, Naim HY et al.: Deglycosylation by small intestinal epithelial cell betaglucosidases is a critical step in the absorption and metabolism of dietary flavonoid glycosides in humans. Eur J Nutr 2003, 42:29-42

50. Walgren RA, Karnaky KJ, Jr., Lindenmayer GE, Walle T: Efflux of dietary flavonoid quercetin 4'-beta-glucoside across human intestinal caco-2 cell monolayers by apical multidrug resistance-associated protein-2. J Pharmacol Exp Ther 2000, 294:830-6.

51. Shirai M, Moon JH, Tsushida T, Terao J: Inhibitory effect of a quercetin metabolite, quercetin 3-o-beta-d-glucuronide, on lipid peroxidation in liposomal membranes. J Agric Food Chem 2001, 49:5602-8.

52. Uekusa $Y$, Kamihira-Ishijima $M$, Sugimoto $O$, Ishii $T$, Kumazawa S, Nakamura K et al.: Interaction of epicatechin gallate with phospholipid membranes as revealed by solidstate nmr spectroscopy. Biochim Biophys Acta 2011, 1808:1654-60.

53. Passamonti S, Terdoslavich M, Franca R, Vanzo A, Tramer F, Braidot $E$ et al.: Bioavailability of flavonoids: A review of their membrane transport and the function of bilitranslocase in animal and plant organisms. Curr Drug Metab 2009, 10:369-394.

54. Piskula MK, Murota K, Terao J: Bioavailability of flavonols and flavones. In Flavonoids and related compounds: Bioavailability and function. Edited by Spencer JP and Crozier A. Boca Raton, FL: CRC Press; 2012:93-107.

55. van der Woude $H$, Boersma MG, Vervoort J, Rietjens IM: Identification of 14 quercetin phase ii mono- and mixed conjugates and their formation by rat and human phase ii in vitro model systems. Chem Res Toxicol 2004, 17:1520-30.

56. Piskula MK, Terao J: Accumulation of (-)-epicatechin metabolites in rat plasma after oral administration and distribution of conjugation enzymes in rat tissues. J Nutr $1998,128: 1172-8$
57. Murota K, Nakamura Y, Uehara M: Flavonoid metabolism: The interaction of metabolites and gut microbiota. Biosci Biotechnol Biochem 2018, 82:600-610.

58. Chen J, Wang S, Jia X, Bajimaya S, Lin H, Tam VH et al.: Disposition of flavonoids via recycling: Comparison of intestinal versus hepatic disposition. Drug Metab Dispos 2005, 33:1777-84.

59. Adlercreutz H, Höckerstedt K, Bannwart C, Bloigu S, Hämäläinen E, Fotsis T et al.: Effect of dietary components, including lignans and phytoestrogens, on enterohepatic circulation and liver metabolism of estrogens and on sex hormone binding globulin (shbg). J Steroid Biochem 1987, 27:1135-1144.

60. Ikeda I, Sasaki E, Yasunami H, Nomiyama S, Nakayama M, Sugano $M$ et al.: Digestion and lymphatic transport of eicosapentaenoic and docosahexaenoic acids given in the form of triacylglycerol, free acid and ethyl ester in rats. Biochimica et biophysica acta 1995, 1259:297-304.

61. Murota K, Takagi M, Watanabe Y, Tokumura A, Ohkubo T: Roe-derived phospholipid administration enhances lymphatic docosahexaenoic acid-containing phospholipid absorption in unanesthetized rats. Prostaglandins Leukot Essent Fatty Acids 2018, 139:40-48.

62. Tomonaga N, Tsuduki T, Manabe $\mathrm{Y}$, Sugawara T: Sphingoid bases of dietary ceramide 2-aminoethylphosphonate, a marine sphingolipid, absorb into lymph in rats. Journal of lipid research 2019, 60:333-340.

63. Matern H, Matern S: Formation of bile acid glucosides and dolichyl phosphoglucose by microsomal glucosyltransferases in liver, kidney and intestine of man. Biochimica et Biophysica Acta (BBA) - Lipids and Lipid Metabolism 1987, 921:1-6.

64. Chen IL, Tsai YJ, Huang CM, Tsai TH: Lymphatic absorption of quercetin and rutin in rat and their pharmacokinetics in systemic plasma. J Agric Food Chem 2010, 58:546-51.

65. Rothwell JA, Day AJ, Morgan MR: Experimental determination of octanol-water partition coefficients of quercetin and related flavonoids. J Agric Food Chem 2005, 


\section{$53: 4355-4360$}

66. Murota K, Shimizu S, Chujo H, Moon JH, Terao J: Efficiency of absorption and metabolic conversion of quercetin and its glucosides in human intestinal cell line caco-2. Arch Biochem Biophys 2000, 384:391-397.

67. Azuma K, Ippoushi K, Ito H, Higashio H, Terao J: Combination of lipids and emulsifiers enhances the absorption of orally administered quercetin in rats. J Agric Food Chem 2002, 50:1706-1712.

68. Azuma K, Ippoushi K, Ito H, Horie H, Terao J: Enhancing effect of lipids and emulsifiers on the accumulation of quercetin metabolites in blood plasma after the short-term ingestion of onion by rats. Biosci Biotechnol Biochem 2003, 67:25482555.

69. Lesser S, Cermak R, Wolffram S: Bioavailability of quercetin in pigs is influenced by the dietary fat content. J Nutr 2004, 134:1508-1511.

70. Lesser S, Cermak R, Wolffram S: The fatty acid pattern of dietary fat influences the oral bioavailability of the flavonol quercetin in pigs. Br J Nutr 2006, 96:1047-1052.

71. Murota K, Hotta A, Ido H, Kawai Y, Moon JH, Sekido K et al.: Antioxidant capacity of albumin-bound quercetin metabolites after onion consumption in humans. J Med Invest 2007, 54:370-374

72. Shirai M, Yamanishi R, Moon JH, Murota K, Terao J: Effect of quercetin and its conjugated metabolite on the hydrogen peroxide-induced intracellular production of reactive oxygen species in mouse fibroblasts. Biosci Biotechnol Biochem 2002, 66:1015-1021.

73. Labrid C: A lymphatic function of daflon $500 \mathrm{mg}$. Int Angio $1995,14: 36-8$

74. Makino R, Takano K, Kita K, Nishimukai M: Influence of longterm feeding of high-fat diet on quercetin and fat absorption from the small intestine in lymph duct-cannulated rats. Biosci Biotechnol Biochem 2018, 82:2007-2011.

75. Wu H, Zhou A, Lu C, Wang L: Examination of lymphatic transport of puerarin in unconscious lymph duct-cannulated rats after administration in microemulsion drug delivery systems. Eur J Pharm Sci 2011, 42:348-53.

76. Zhou A, Lu T, Wang L, Lu C, Wang L, Wan M et al.: Lymphatic transport of puerarin occurs after oral administration of different lipid-based formulations to unconscious lymph duct-cannulated rats. Pharm Dev Technol 2014, 19:743-7.

77. Xu Q, Zhou A, Wu H, Bi Y: Development and in vivo evaluation of baicalin-loaded w/o nanoemulsion for lymphatic absorption. Pharm Dev Technol 2019, 24:11551163.

78. Yao M, Chen J, Zheng J, Song M, McClements DJ, Xiao H: Enhanced lymphatic transport of bioactive lipids: Cell culture study of polymethoxyflavone incorporation into chylomicrons. Food Funct 2013, 4:1662-7.

79. Mukai R, Fujikura Y, Murota K, Uehara M, Minekawa S, Matsui $\mathrm{N}$ et al.: Prenylation enhances quercetin uptake and reduces efflux in caco-2 cells and enhances tissue accumulation in mice fed long-term. J Nutr 2013, 143:155864.

80. Shimoi K, Saka N, Nozawa R, Sato M, Amano I, Nakayama T et al.: Deglucuronidation of a flavonoid, luteolin monoglucuronide, during inflammation. Drug Metab Dispos 2001, 29:1521-1524.

81. Kawai Y, Nishikawa T, Shiba Y, Saito S, Murota K, Shibata N et al.: Macrophage as a target of quercetin glucuronides in human atherosclerotic arteries: Implication in the antiatherosclerotic mechanism of dietary flavonoids. J Biol Chem 2008, 283:9424-9434.

82. Kawai Y: Beta-glucuronidase activity and mitochondrial dysfunction: The sites where flavonoid glucuronides act as anti-inflammatory agents. J Clin Biochem Nutr 2014, 54:14550.

83. Martínez G, Mijares MR, De Sanctis JB: Effects of flavonoids and its derivatives on immune cell responses. Recent Pat Inflamm Allergy Drug Discov 2019, 13:84-104.

84. Jafarinia M, Sadat Hosseini M, Kasiri N, Fazel N, Fathi F, Ganjalikhani Hakemi M et al.: Quercetin with the potential effect on allergic diseases. Allergy Asthma Clin Immunol 2020, 16:36. 
85. Jiang M, Li Z, Zhu G: Immunological regulatory effect of flavonoid baicalin on innate immune toll-like receptors. Pharmacol Res 2020, 158:104890.

86. Bentsath A, Rusznyak ST, Szent-Gyorgyi A: Vitamin nature of flavones. Nature 1936, 138:798-798. 